\title{
Impact of Antenna Pattern and Link Model on Directional Neighbor Discovery in $60 \mathrm{GHz}$ Networks
}

\author{
Xueli An, R. Venkatesha Prasad, and Ignas Niemegeers \\ Faculty of EEMCS, Delft University of Technology, The Netherlands \\ Email: $\{$ X.An, R.R.VenkateshaPrasad, I.G.M.M.Niemegeers $\} @$ tudelft.nl
}

\begin{abstract}
GHz radio is a very attractive technology for short-range wireless communication due to its capability to provide Gbps data rate. To address the neighbor discovery (ND) problem in $60 \mathrm{GHz}$ networks, we propose a novel analytical framework to investigate the ND performance. The main difficulty in modeling the ND process in a $60 \mathrm{GHz}$ network is the involvement of the directional antennas with gain differences between the antenna's main lobe and side lobes. Different antenna modes - directional or omni-directional - coupled with different ND mechanisms make the analytical study demanding. In this article, we propose a comprehensive theoretical model to demonstrate the performance of ND processes using one-way ND and handshake-based ND mechanisms. Moreover, we combine them with different antenna modes, i.e., directional transmitting with omni-directional listening (DO) and directional transmitting with directional listening (DD) modes. The impact of antenna modes on the ND process is analyzed. Since $60 \mathrm{GHz}$ radio is prone to co-channel interference, we examine a realistic interferenceaware link model and antenna pattern via simulation studies. Our work is specifically beneficial to provide guidelines for applying directional neighbor discovery process within $60 \mathrm{GHz}$ wireless networks.
\end{abstract}

Index Terms-Neighbor discovery, $60 \mathrm{GHz}$ radio, directional antennas, performance analysis

\section{INTRODUCTION}

To cater to the emerging wireless multimedia applications like uncompressed HD video streaming in indoor environments, data rates of the order of gigabits per second (Gbps) are required. This requirement is difficult to achieve with the current wireless technologies, such as, IEEE 802.11x WLAN. Therefore, the current focus of many research initiatives is to exploit the recently opened $60 \mathrm{GHz}$ frequency band. Until now, multi-GHz bandwidth has been allocated worldwide in the $60 \mathrm{GHz}$ spectrum, and it is capable of supporting Gbps-based wireless communication. In March 2005, the IEEE 802.15.3 Task Group 3c (TG3c) [1] was formed to develop a $60 \mathrm{GHz}$ based alternative physical layer for the existing IEEE 802.15.3 standard to enable Gbps-based wireless personal area networks (WPANs). It was approved as a standard in September 2009. While trying to use $60 \mathrm{GHz}$ radio for short-range highspeed wireless communication, we have to face many special challenges while designing higher layers protocols due to its unique properties. For instance, the $60 \mathrm{GHz}$ radio suffers from high path loss. To obtain sufficient link budget for multi-Gbps data rates, directional antennas are adopted in $60 \mathrm{GHz}$ systems. Especially, for the adaptive array-based directional antenna, its capability of beam-forming provides the flexibility to align the antenna's transmitting and receiving directions [2]. Directional antennas are not only applicable for $60 \mathrm{GHz}$ systems, but are also widely used in wireless systems to increase link gain and reduce the interference area [3]-[6]. Although directional antennas offer many advantages over omni-directional antennas, their deployment is highly challenging for WPANs.

For a certain device, all the devices that are within its transmission range can be considered as its neighbors. A neighbor discovery (ND) process allows in-range devices to detect each other and establish links to form a connected network. Wireless ad-hoc networks are envisioned to be selforganized, which means devices are expected to set-up and maintain networks without relying on any external infrastructure, or human interventions. Hence, ND is an essential process to realize self-organization in wireless ad-hoc networks [7]. It cooperates with the medium access, service discovery, and routing protocols that require specific information about neighbors. The required duration and efficiency of a ND process directly affects the network setup time, time to find routes, etc. ND protocols can be generally classified as direct ND protocols and gossip-based ND protocols [8]. During a ND process, devices broadcast their advertisement messages, which are the so called HELLO messages. If a device directly receives an advertisement message from another device, then they are one-hop neighbors. When a direct ND protocol is used, a device only discovers its neighbors by directly receiving their advertisement messages. When a gossip-based ND protocol is used, devices not only broadcast their own advertisement messages, but also the information about their neighbors to speed up the ND process. Hitherto, omni-directional antennas are widely addressed in ND protocols [9], [10]. A ND process using omni-directional is quite straightforward. Once a device accesses the channel and broadcasts its advertisement message, all the devices that are in the transmission range of the device may receive its advertisement message. If multiple advertisement messages arrive at the receiver simultaneously, none of them can be received because of the collisions of the advertisement messages.

To set-up a directional communication, a device not only needs to know who its neighbor are, but it also requires the information that where the neighbors are. Hence, it is necessary for devices to determine the direction of each other before setting up directional communications. Within 
the indoor environment, there are normally more than one beam paths that can be used for communication - one lineof-sight (LOS) path and multiple non-LOS (NLOS) paths. Since $60 \mathrm{GHz}$ radio is very prone to the channel variation and LOS blockage phenomenon [11], devices need to trace the best beam path that could provide best quality during the ND process. Hence, gossip-based ND protocols are not suitable in this case. Therefore, we address direct ND protocols in detail. According to the reply mechanism used in a ND process, direct ND protocols can be further classified as one-way $N D$ and handshake based ND [12]:

- One-way ND: In one-way ND protocols, each device periodically transmits advertisement messages to announce its presence, and discovers its neighbors by receiving similar advertisement messages from other devices.

- Handshake based ND: In handshake based ND protocols, once a device receives an advertisement message, it provides active response to its neighbor. Compared to one-way ND protocols, handshake based ND protocols are more complex to implement, but they are easy to construct symmetric neighborhood by exchanging advertisement messages especially for direction-aware links.

Although the directional antennas offer many advantages over omni-directional antennas, their deployment for neighbor discovery is not trivial. For instance, deafness is a typical problem caused while using directional antennas [6]. The performance of a directional ND (D-ND) process might be affected by deafness phenomenon when a device, say $A$, fails to communicate with the other device $B$, because device $B$ points its antenna main lobe in a direction which is away from $A$. A device can also nullify the interference from undesired directions using directional antennas for receiving, which may help to alleviate the collisions of advertisement messages. Therefore, it is very interesting to know the exact influence of using directional antennas in a ND process. To achieve the best link quality, devices need to find out the best beam path to communicate with its neighbors. Therefore, it is preferred that Directional Advertisement (DA) messages are to be transmitted in a blind way. Here blind means that devices sweep the DA messages in all the possible directions, which helps devices to update the beam path status of their neighbors in time. According to the mechanisms used to transmit the DA messages, D-ND protocols can be executed in two ways: randomized $D-N D$ or scanning-based $D-N D$. For a randomized D-ND process, devices randomly pick a direction to transmit their DA messages once they have access to the channel. Vasudevan, et al., presented several probabilistic models for D-ND protocols in [8], but their approach is only applicable to one-way randomized D-ND processes. In a scanning-based D-ND process ${ }^{1}$, if a device is in the transmitting state, it randomly selects a beam sector to transmit its DA messages, and moves (counter-)clockwise to transmit the next DA message in the next sector until it covers all the beam sectors. Zhang, et al., proposed a simplistic

\footnotetext{
${ }^{1}$ The antenna system we considered here is adaptive array based antenna system which is capable of beam-forming and its antenna main lobe is electronically steerable [2].
}

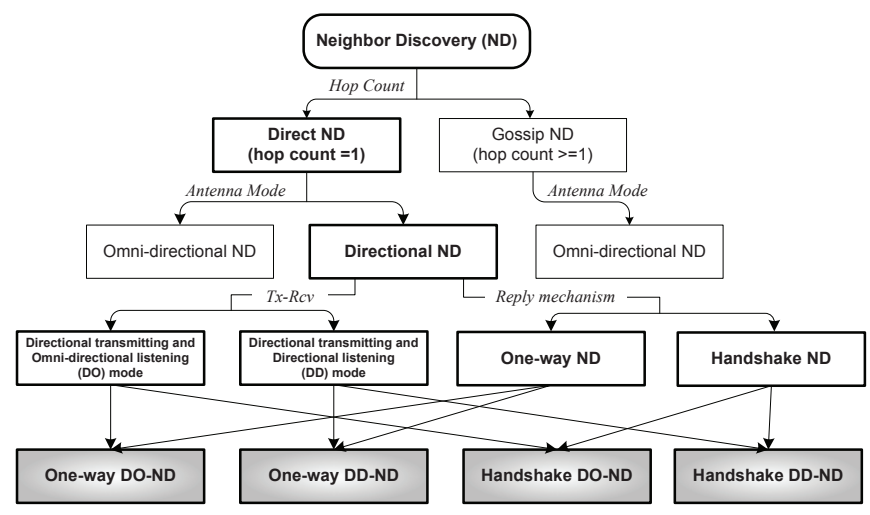

Fig. 1. Neighbor discovery protocol classification

analytical model for handshake and scanning-based D-ND in [12]. They assumed that in each beam sector only one potential neighbor is present, which makes the analysis only applicable to sparse networks with narrow beam antennas. The one-way and scanning-based D-ND process is not investigated in their work. D-ND in $60 \mathrm{GHz}$ indoor wireless networks was thoroughly investigated in [13]. The method used there is also based on one-way randomized D-ND mechanism. Moreover, they assumed that reception failure is only caused by packet collisions, in which the effect of the channel is not considered. Neighbor location discovery via direct path or non-direct path using linear and circular polarization and different responses to reflections with directional antennas operating in the $60 \mathrm{GHz}$ band was emphasized in [14].

To provide a comprehensive view of the scanning-based D-ND protocols, we are motivated to study the one-way and handshake-based ND protocols combining with different antenna modes used for transmission and reception, i.e., with directional transmission and omni-directional listening (DO) and directional transmission and directional listening (DD) modes. Figure 1 provides a detailed classification of ND protocols and also positions our work with respect to many ND processes under this classification.

The main contribution of this work is two-fold: (1) We propose an in-depth analytical model which can be used to demonstrate the performance of D-ND protocols in terms of the percentage of the detected neighbors in one ND process. Furthermore, the required duration for this ND process is also modeled. We study the performance of D-ND protocols in two scenarios. In the first scenario, we investigate the performance of a peer-to-peer (two-device) discovery process using directional antennas. In the second scenario, we investigate the D-ND protocols in a distributed ad-hoc network to provide a generic case with multiple devices. The accuracy of our theoretical model is validated with extensive simulations. However, due to the complexity in framing an exact analytical model, we deduced our model based on certain assumptions and constraints. For instance, we assumed an idealized antenna model and full mesh network. (2) Hence, the second contribution of this work is that, we revisit our model and find the influence of the antenna pattern and the link model on the DND processes using simulations. We provide a detailed view 
of ND process using $60 \mathrm{GHz}$ radio, the ND performance was studied using a Uniform Circular Array (UCA) system with an interference-aware link model. To the best of our knowledge, this work is the first one that provides an in-depth investigation on the D-ND performance in $60 \mathrm{GHz}$-based wireless networks considering both impact of the antenna pattern and the link model at the same time.

The rest of this article is organized as follows. In Section II, we introduce our system model and list all the assumptions used in this work. In Section III, we present two antenna patterns, based on which we discuss the influence of the radio link model on the ND process. In Section IV, we provide a theoretical model for the various D-ND processes for a specific two-device model and also a generic model with multiple devices. The accuracy of the analytical model is validated via simulations in Section V. In Section VI, we discuss the assumptions used in the analytical work and compare them with the realistic antenna pattern and link model via extensive simulations. Finally, we conclude our work in Section VII.

\section{System Model And Assumptions}

In this work we assume two-dimensional network. All the devices are located on the same horizontal plane. The halfpower beamwidth ( $3 \mathrm{~dB}$ beamwidth) of directional antennas, which is also called as antenna beamwidth, is denoted by $\theta_{b}$. According to the antenna beamwidth, the area around a device is divided into $N_{b}$ equal sectors, where $N_{b}=\frac{2 \pi}{\theta_{b}}$. A slotted system is assumed in our analytical model, in which, all the devices are synchronized together. Synchronization is not difficult to achieve in the centrally controlled WPAN, which relies on a piconet controller (PNC) to provide the timing information for all the devices within the WPAN [15]. The duration of each time slot, $\tau$, is equal to the transmission time of a DA message. The scanning-based mechanism is adopted in this work for D-ND. For one-way D-ND, every $N_{b}$ slots are grouped together as a frame. The time duration of a frame, which is also considered as the duration for one time of scanning, is $\tau N_{b}$. For handshake based D-ND, the duration of each handshake process is defined as the duration for transmitting a DA message and a DA Acknowledgment (ACK) message from the receiver. We assume that a DA ACK also contains the DA message from the receiver. Every $2 N_{b}$ slots are grouped together as a frame, and the length of a frame is equal to $2 \tau N_{b}$, as shown in Fig. 2.

A device is either in the transmitting state or listening state. The medium access mechanism assumed in our ND protocol is contention-based. At the beginning of each frame, a device has a probability $p_{t}$ to be in the transmitting state or has a probability $1-p_{t}$ to be in the listening state. Contentionbased medium access is also compatible with the IEEE 802.15.3c standard. For the IEEE 802.15.3c MAC, it inherits the IEEE 802.15.3 MAC which contains contention-based medium access and contention-free medium access. The main network components within a WPAN are personal electronic devices like laptops, PDAs, etc. Hence, a contention-based medium access for a ND process provides more freedom for the devices for self-advertising and network information

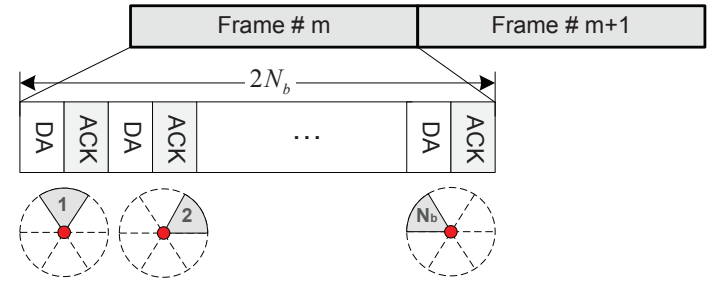

Fig. 2. Frame structure for handshake-based neighbor discovery process using directional antennas.

detection and upgrade. To investigate the influence of using directional antennas in ND process we define that all the ND-related packets, i.e., DAs and DA ACKs, are transmitted using directional mode. According to the antenna mode used in the listening state, ND processes can be classified into two categories: directional transmitting and omni-directional listening (DO) mode, directional transmitting and directional listening (DD) mode. Once a device is in the directional listening state, it randomly picks up a beam sector to listen and listens in the same direction for the entire frame. According to the combination of ND protocols and antenna modes, there are four D-ND protocols that are examined in the sequel: oneway DO-ND, one-way DD-ND, handshake based DO-ND and handshake based DD-ND, as shown in Fig. 1.

To quantify the performance of a ND process, we first define the following metrics.

- ND ratio is defined as the ratio between the number of discovered neighbors to the number of all the surrounding neighbors. The ND ratio determines the network topology and robustness. A higher ND ratio means a better connectivity in the entire network.

- ND time is an important metric to characterize the duration of one ND process. It can be viewed as the time spent to let any newly entering device to discover and incorporate with all or most of the neighboring devices. The number of required slots for a ND process can be used as an indicator of ND time. We assume that a ND process is considered as complete if the ND ratio reaches $99 \%$. This metric gives an indication as to how effective the ND process is.

- ND overhead is the number of generated DA and DA ACK messages during the ND process. This metric gives an indication as to how efficient the ND process is.

\section{Influence of Antenna Pattern AND Link Model ON THE ND PROCESS}

The radio link is prone to the variations in channel quality. Especially for $60 \mathrm{GHz}$ radio, a small scale signal strength variation might influence the connectivity between two devices even when they are close by. Moreover, the imperfections in antenna patterns with side lobe effects might also impact the ND performance. To get a better understanding of the D-ND process in a realistic scheme, we first introduce several antenna patterns and link models in this section. 


\section{A. Directional Antenna}

Adaptive array based directional antenna systems, also known as smart antennas, are composed of a number of antenna elements. The antenna radiation pattern is controlled by the amplitude and phase weights of each individual element. The weights could be varied with time. The main lobe of an adaptive array system is steerable in different directions, which is called antenna beam-forming. It is achieved by adaptively adjusting the weight of each antenna element to locate and track the received signals. According to the input from each element, the Direction of Arrival (DoA) of the captured signal is computed using some algorithms, e.g., MUSIC [16] and ESPRIT [17]. The DoA information can be cached during the ND process.

1) Idealized Flat-Top Antenna System: An idealized directional antenna pattern with neglected side lobe is introduced here, which is called "flat-top" antenna system. The antenna gain of the flat-top antenna system can be denoted as $G=10 \log \left(2 \pi / \theta_{b}\right)$. Hence, if the antenna main lobe is directed in the direction $\theta_{s}$, the antenna gain within the range $\left[\theta_{s}-\theta_{b} / 2, \theta_{s}+\theta_{b} / 2\right]$ is $G$, and the antenna gain outside this range is assumed to be zero. Because flat-top is an idealized antenna pattern, it provides an easy way to model the behavior and influence of directional antennas. It is widely used in the analysis involving directional antennas, see [8] and [12].

2) Uniform Circular Array Antenna System: Uniform circular array (UCA) antenna system is a specific case of the adaptive array antenna. For a UCA antenna system, the antenna elements are uniformly arranged on a circular disc in the $x-y$ plane. A brief deduction of the antenna gain pattern of a UCA system with $N$ identical antenna elements is provided here for the sake of completeness [18]. If each element has the same magnitude, the array factor of a UCA system can be expressed as

$$
(A F)_{n}=\left|J_{0}(k \rho)+2 \sum_{m=1}^{\infty}(j)^{m N} J_{m N}(k \rho) \cos (m N \xi)\right|,
$$

where,

$$
\begin{aligned}
\xi & =\tan ^{-1}\left[\frac{\sin \theta \sin \phi-\sin \theta_{0} \sin \phi_{0}}{\sin \theta \cos \phi-\sin \theta_{0} \cos \phi_{0}}\right] \\
\rho & =R\left[\left(\sin \theta \cos \phi-\sin \theta_{0} \cos \phi_{0}\right)^{2}\right. \\
& \left.+\left(\sin \theta \sin \phi-\sin \theta_{0} \sin \phi_{0}\right)^{2}\right]^{\frac{1}{2}} .
\end{aligned}
$$

The term $J_{m}(x)$ above is the Bessel function of the first kind, $J_{0}(k \rho)$ is the principal term and the summation part is the residual term. The antenna gain is given by

$$
\begin{aligned}
& G(\theta, \phi)=\eta \frac{U(\theta, \phi)}{\frac{1}{4 \pi} \int_{0}^{2 \pi} \int_{0}^{\pi} U(\theta, \phi) \sin (\theta) \mathrm{d} \theta \mathrm{d} \phi} \\
& =4 \pi\left[\left|J_{o}(k \rho)+2 \sum_{m=1}^{\infty}(j)^{m N} J_{m N}(k \rho) \cos (m N \xi)\right|\right]^{2} \\
& \times\left[\int _ { 0 } ^ { 2 \pi } \int _ { 0 } ^ { \pi } \left(\mid J_{o}(k \rho)+2 \sum_{m=1}^{\infty}(j)^{m N} J_{m N}(k \rho)\right.\right. \\
& \left.\cos (m N \xi) \mid)^{2} \times \sin \theta \mathrm{d} \theta \mathrm{d} \phi\right]^{-1}
\end{aligned}
$$

where, $\eta$ is the dimensionless term which represents antenna radiation efficiency. According to (2), the antenna gain and
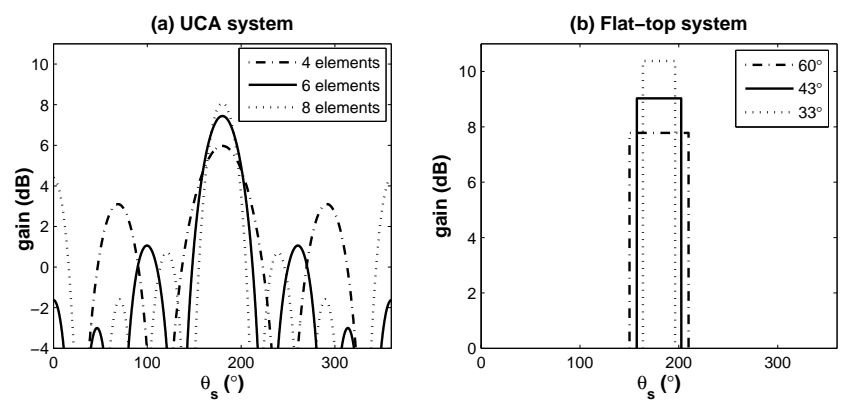

Fig. 3. Antenna gain pattern for different antenna elements. For the UCA system, $\theta$ is set as $\pi / 2$ to only observe the $x-y$ plane. The space between each adjacent elements is set as half of the radio wavelength, $d_{n}=\lambda / 2$, $\eta=1$. The main beam directed at $\theta_{0}=\pi / 2, \phi_{0}=0$.

TABLE I

UCA RELATED PARAMETERS, WITH $d_{n}=\frac{\lambda}{2}, \eta=1$

\begin{tabular}{c|c|c|c}
\hline \hline Num. of elements & Radius & Gain $(\mathrm{dB})$ & $\theta_{H P B W}$ at x-y plane $\left(^{\circ}\right)$ \\
\hline 4 & $0.35 \lambda$ & 5.97 & 63.24 \\
6 & $0.50 \lambda$ & 7.44 & 43.76 \\
8 & $0.65 \lambda$ & 8.08 & 33.01 \\
\hline \hline
\end{tabular}

antenna beamwidth obtained using 4, 6, 8 elements UCA systems are listed in Table I, which is found by setting $\theta$ as $\pi / 2$ to only observe the $x-y$ plane. The space between each adjacent element, $d_{n}$, is set as half of the radio wavelength, $d_{n}=\lambda / 2$. The main beam is directed at $\theta_{0}=\pi / 2, \phi_{0}=0$, and $\eta=1$. The gain patterns of UCA antenna systems are shown in Fig. 3 (a) with 4, 6 and 8 elements. To enable comparison with the UCA systems, the flat-top antennas are selected with the similar beamwidth as that of UCA systems and their gain patterns are shown in Fig. 3 (b).

\section{B. Radio Propagation Model and Link Availability}

As we know that the received signal power over the wireless channel at the receiver side decreases as the distance between the transmitter and the receiver increases, which is called as path loss. We consider path loss represented using a lognormal shadowing radio propagation model which is given by [19],

$$
P_{r}(d)=P_{t}+G_{t}+G_{r}-I_{L}-\left(P L_{0}+10 n \log (d)+X_{\sigma}\right),
$$

in which, $P_{t}$ is the transmitting power, $G_{t}$ and $G_{r}$ are the antenna gains of the transmitter and receiver, respectively. $I_{L}$ is the implementation loss, which is caused by various circuit impairments. $P L_{0}$ is the reference path loss at 1 meter and $d$ is the transmission distance. Parameter $n$ is the path loss exponent. The measured path loss exponent for wide-band (e.g. $1 \mathrm{GHz}$ ) radio is close to 2 under LOS conditions with directional antennas [20] [21]. $X_{\sigma}$ is a zero mean Gaussian random variable with a standard deviation, $\sigma$.

1) Link Model 1 (LM1): To correctly demodulate and decode transmitted signals, the received signal power should be higher than a certain threshold, $\gamma_{t h}$, which is also called receiving sensitivity. Therefore, the coverage range of a device refers to the area in which the received signal strength from the device is higher than the threshold $\gamma_{t h}$. Without considering 
the shadowing effect $(\sigma=0)$, a maximum transmission range $r_{t h}$ can be calculated as $r_{t h}=10^{\frac{\kappa}{10 n}}$, where $\kappa=$ $P_{t}+G_{t}+G_{r}-I_{L}-P L_{0}-\gamma_{t h}$. In LM1 we define that all the devices within the radius $r_{t h}$ from the device are considered as its direct neighbors. The probability of existence of a link between a transmission pair $i$ and $j$ is denoted as $p_{i j}$, which is also called as link availability or link probability in the rest of this article. Hence we represent it as a simple step function

$$
p_{i j}= \begin{cases}1 & 0<\|i-j\| \leq r_{t h} \\ 0 & \text { otherwise }\end{cases}
$$

where, $\|i-j\|$ is the Euclidean distance between $i$ and $j$.

2) Link Model 2 (LM2): As shown in Fig. 3 (a), the antenna side lobes play an important role in a UCA system. They may introduce interference to other transmissions, or be used for communication. Hence a more realistic link model is used in LM2 considering the interference from other transmitters. To guarantee a required transmission performance for a transmission pair $(i, j)$, the Signal to Interference-plusNoise Ratio (SINR) should be higher than a certain threshold $\varphi_{t h}$. Therefore, a link exists with a probability,

$$
p_{i j}= \begin{cases}1 & \mathcal{P}>\varphi_{t h} \\ 0 & \text { otherwise }\end{cases}
$$

where

$$
\mathcal{P}=10 \log \frac{\mathbb{P}_{r}(\|i-j\|)}{N_{0}+\sum_{k \neq i} \mathbb{P}_{r}(\|k-j\|)}
$$

The term $\mathbb{P}_{r}$ is the received power (in watts), $N_{0}$ is the mean noise power (in watts) and $\sum_{k \neq i} \mathbb{P}_{r}(\|k-j\|)$ is the accumulated interference power from the other interfering sources.

3) Link Model 2 Plus shadowing effect (LM2+): According to (3), a received power $P_{r}$ (in $\mathrm{dB}$ ) obtained using lognormal radio propagation model can be represented as a Gaussian distributed variable $P_{r}(d) \backsim \mathcal{N}(\mu, \sigma)$, where $\mu=$ $P_{t}+G_{t}+G_{r}-I_{L}-\left(P L_{0}+10 n \log (d)\right)$. Therefore, the received power $\mathbb{P}_{r}$ (in watts) can be represented as a lognormal variable with mean $\omega$ and standard deviation $\nu$, where $\omega=\exp \left(\mu+\sigma^{2} / 2\right)$ and $\nu=\left(\exp \left(\sigma^{2}\right)-1\right) \exp \left(2 \mu+\sigma^{2}\right)$. Assume that there are $K$ interfering sources. The accumulated interference is represented as the summation of the $K \log$ normally distributed interfering powers, which is very complex to express [22], but it can be approximated as a log-normally distributed variable $\mathbb{L} \backsim \log -\mathcal{N}\left(\mu^{\prime}, \sigma^{\prime}\right)$ according to [23]. Using Fenton-Wilkinson (FW) approximation model [24] ${ }^{2}$, we can have $\mu^{\prime}=2 \ln u_{1}-\frac{1}{2} \ln u_{2}$ and $\sigma^{\prime}=(\ln u 2-2 \ln u 1)^{\frac{1}{2}}$, where $u_{1}=\sum_{i=1}^{K} \exp \left(\omega_{i}+\nu_{i}^{2} / 2\right)$ and $u_{2}=\sum_{i=1}^{K} \exp \left(2 \omega_{i}+\right.$ $\left.2 \nu_{i}^{2}\right)+2 \sum_{i=1}^{K-1} \sum_{j=i-1}^{K} \exp \left(\omega_{i}+\omega_{j}\right) \exp \left(\frac{1}{2}\left(\nu_{i}^{2}+\nu_{j}^{2}\right)\right)$. Compared to the interference from other sources, the noise $N_{0}$ can be neglected. We denote the received signal power from the desired transmitter as $\mathcal{N}\left(\mu^{\prime \prime}, \sigma^{\prime \prime}\right)$. Hence, $\Delta \mathcal{P}$, where $\Delta \mathcal{P}=\mathcal{P}-\varphi_{t h}$, can be expressed as a normally distributed variable $\Delta \mathcal{P} \backsim \mathcal{N}\left(\mu_{\varphi}, \sigma_{\varphi}\right)$, where $\mu_{\varphi}=\mu^{\prime \prime}-\mu^{\prime}-\varphi_{t h}$ and

\footnotetext{
${ }^{2} \mathrm{FW}$ model has a good accuracy when the standard deviation of log-normal components are less than $4 \mathrm{~dB}$ [22], and the measured $\sigma$ for a $60 \mathrm{GHz}$ system in LOS scenario is smaller than $4 \mathrm{~dB}$ [19].
}
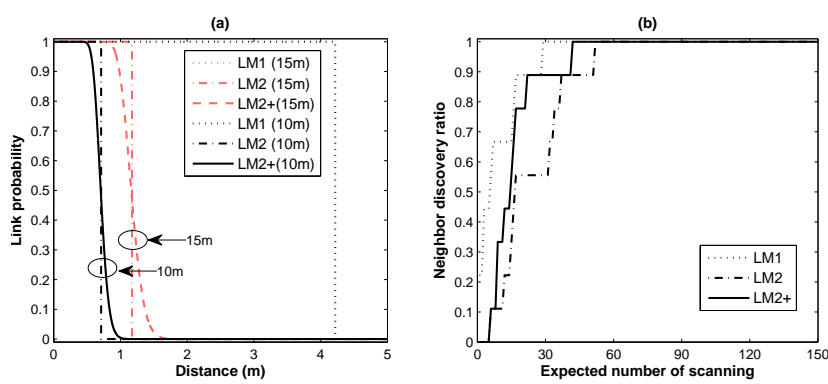

Fig. 4. Influence of link model on link probability and ND ratio using $60 \mathrm{GHz}$ radio with $1 \mathrm{GHz}$ bandwidth, $P L_{0}=68 \mathrm{~dB}, I_{L}=1.5 \mathrm{~dB}$, receiving sensitivity $-72 \mathrm{dBm}$, SINR threshold $12 \mathrm{dBm}, n=2, \sigma=1$. (a) 5 interfering nodes, network radius $10 \mathrm{~m}$ and $15 \mathrm{~m}, G_{t}=G_{r}=0$. (b) 6 elements UCA system, node degree is 10 , network radius $5 \mathrm{~m}, p_{t}=0.2$.

$\sigma_{\varphi}^{2}=\sigma^{\prime \prime 2}+\sigma^{\prime 2}$. Hence the link probability between $i$ and $j$ considering interference is given by [25],

$$
\begin{aligned}
p_{i j} & =\operatorname{Pr}[\Delta \mathcal{P}>0]=\frac{1}{\sigma_{\varphi} \sqrt{2 \pi}} \int_{0}^{\infty} \exp \left[-\frac{t-\mu_{\varphi}}{2 \sigma_{\varphi}^{2}}\right] d t \\
& =\frac{1}{2}\left(1-\operatorname{erf}\left(\frac{-\mu_{\varphi}}{\sqrt{2} \sigma_{\varphi}}\right)\right) .
\end{aligned}
$$

4) Discussion: To demonstrate the impact of link models on the link probability, five transmitters are uniformly distributed within a circular network of radius $10 \mathrm{~m}$ and $15 \mathrm{~m}$, in which a target device is at the center of the network. Without considering the impact of directional antennas, the antenna gains are set to zero $\mathrm{dB}$. For the target device, the resultant link probability plots using different link models are shown in Fig. 4 (a). The link budget related parameter settings are listed in the caption of Fig. 4. Since $60 \mathrm{GHz}$ radio is very fragile to co-channel interference, the maximum transmission range that is obtained using LM1 is much longer than the achievable transmission range with the existence of interference. The link probability using LM2+ considering log-normal radio propagation model indicates that a slight variation in distance may lead to connectivity failure for $60 \mathrm{GHz}$ links.

To obtain some basic idea about the influence of the adopted LM on the performance of ND process, we study a scenario through simulations. The set up is as follows: ten devices are uniformly distributed within a circular network of radius $5 \mathrm{~m}$, and the target device is at the center of the network. Each device is equipped with 6 elements UCA system. The transmission probability is set to 0.2 . The ND ratios using different LMs after one-way DO-ND process are depicted in Fig. 4 (b), in which LM1, LM2 and LM2+ exhibit different ND performances. A detailed investigation of the influence of link models on the ND process is introduced later in this article.

\section{Analytical Model for Directional Neighbor DISCOVERY}

In this section, a theoretical model is proposed to analyze the performance of D-ND protocols. Our proposed model is a generic model which can be used for any system equipped with directional antennas. Since it is not trivial to model the exact 
properties of realistic antenna patterns, like the UCA system, the flat-top antenna system and LM1 are used in our theoretical model to alleviate the influence of side lobes. Moreover, a full mesh network is assumed, in which all the devices are within the transmission range of each other.

\section{A. D-ND Analysis for Two-Device Model}

The two-device model is a simple and realistic scenario to study a ND process. For instance, when a Blu-ray player sets up connection with a HDTV display for video streaming, or two laptops connect to each other for high speed downloading, the latency of a ND process directly influences the user experience of the consumer products. Therefore, it is necessary to model first the ND performance using directional antennas two devices.

1) One-way $D O-N D$ and $D D-N D$ : In a one-way DO$\mathrm{ND}$, devices transmit with directional antennas, and listen with omni-directional antennas. Let us assume that device $m$ and device $n$ are one-hop neighbors. The condition for $m$ to discover $n$ in a certain frame is that $m$ is in the listening state and $n$ is in the transmitting state, the probability of which is given by $p_{f}=\left(1-p_{t}\right) p_{t}$, where $p_{t}$ is the probability for a device to be in the transmitting state. Assume one of the two devices discovers its neighbor in the $i^{\text {th }}$ frame, and its neighbor discovers it in the $j^{t h}$ frame, where $i<j$. Therefore, this one-way DO-ND process is completed in the $j^{\text {th }}$ frame with probability $p_{1 D O}^{(j)}$, which is given by

$$
p_{1 D O}^{(j)}=2 p_{f}\left(\left(1-p_{f}\right)^{j-1}-\left(1-2 p_{f}\right)^{j-1}\right) .
$$

The probability for the two devices discovering each other within $J$ frames is given as $p_{J}=\sum_{j=2}^{J} p_{1 D O}^{(j)}$. Note that the two devices cannot discover each other within the same frame, therefore, it requires at least two frames. Thus the summation starts from $j=2$. The expected number of slots required for this one-way DO-ND process is given by,

$$
N_{1 D O}=\sum_{j=2}^{J} N_{b} j p_{1 D O}^{(j)}
$$

In the one-way DD-ND case, both transmitting and receiving devices use directional antennas. To discover a neighbor, the receiver needs to point its directional antenna in the direction of the transmitter to receive its DA message. The probability of this event is given by $p_{f}=\frac{1}{N_{b}}\left(1-p_{t}\right) p_{t}$. We denote $p_{1 D D}^{(j)}$ as the probability for the two devices discovering each other in the $j^{\text {th }}$ frame. We use $N_{1 D D}$ as the expected number of slots for the one-way DD-ND process. The two parameters $p_{1 D D}^{(j)}$ and $N_{1 D D}$ can be calculated by substituting $p_{f}$ in (7) and (8).

2) Handshake based $D O-N D$ and $D D-N D$ : In a handshake based mechanism, an active response is provided if a device receives a DA message from the other device. Hence, for the two-device model, it is possible for device $m$ and $n$ discovering each other within the same frame, and this probability is given by $p_{f}=2\left(1-p_{t}\right) p_{t}$. Let us represent the probability that the two devices discover each other in the $j^{\text {th }}$ frame for the first time as $p_{2 D O}^{(j)}$, and it is calculated as,

$$
p_{2 D O}^{(j)}=p_{f}\left(\left(1-p_{f}\right)^{j-1}\right) .
$$
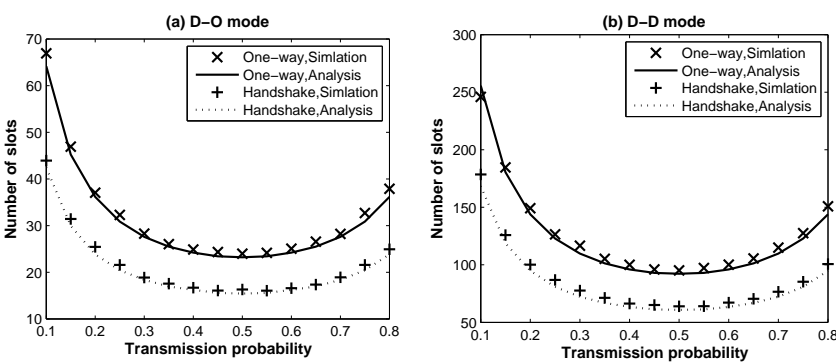

Fig. 5. Neighbor discovery time for two-device model, antenna beamwidth $\theta=60^{\circ}$.

The expected number of slots for a handshake based DO-ND process is expressed as,

$$
N_{2 D O}=\sum_{j=1}^{J} 2 N_{b} j p_{2 D O}^{(j)} .
$$

Based on the above concepts, it is easy to understand that for the handshake based DD-ND, $p_{f}$ is obtained as $p_{f}=$ $\frac{2}{N_{b}}\left(1-p_{t}\right) p_{t}$, and then $p_{2 D D}^{(j)}$ and $N_{2 D D}$ can be obtained by substituting $p_{f}$ into (9) and (10).

Proposition 1: For the two-device model, handshake based D-ND mechanism achieves a faster D-ND process than oneway mechanism in a slotted system.

Proof: We firstly examine the DO-ND case. Based on (8) and (10), the difference in the expected number of slots required for handshake based and one-way DO-ND is expressed as,

$$
\begin{aligned}
& N_{2 D O}-N_{1 D O}=\sum_{j=1}^{J} 2 N_{b} j p_{2 D O}^{(j)}-\sum_{j=2}^{J} N_{b} j p_{1 D O}^{(j)} \\
& =\sum_{j=1}^{J} j\left(3\left(1-2 p_{t}\left(1-p_{t}\right)\right)^{i-1}-\left(1-p_{t}\left(1-p_{t}\right)\right)^{i-1}\right) \\
& \times 2 N_{b} p_{t}\left(1-p_{t}\right)=N_{b}(A+B)
\end{aligned}
$$

where

$$
\begin{aligned}
& A=1-\left(1-p_{t}\left(1-p_{t}\right)\right)^{J-1} \\
& B=3\left(\left(1-p_{t}\left(1-p_{t}\right)\right)^{J-1}-\left(1-2 p_{t}\left(1-p_{t}\right)\right)^{J-1}\right) .
\end{aligned}
$$

Since $A$ and $B$ are positive, we have $N_{2 D O}-N_{1 D O}>0$. Similarly, for the DD-ND case, we can also obtain that $N_{2 D D}-N_{1 D D}>0$.

3) Validation using Simulation: To validate the accuracy of the analytical model, we simulated the four D-ND protocols at the algorithmic level ${ }^{3}$ using Matlab (R2007b). The ND process is defined according to the scenario explained in Section II. The analytical and simulation results are compared in Fig. 5 when the antenna beamwidth is fixed at $60^{\circ}$. The simulation results are based on the average of 10000 iterations. The one-way and handshake based ND mechanisms are compared

\footnotetext{
${ }^{3}$ The physical layer simulation is not considered here, that means, in a certain time slot, if a DA message is transmitted without experiencing any DA collision, it can be received by all the receiving devices in the range with probability 1 .
} 
when they combine with DO mode and DD mode respectively. They are shown in Fig. 5 (a) and (b). The two subplots show the relationship between the expected number of slots required for a ND process and the transmission probability $p_{t}$. It is shown that, there exists an optimal value of $p_{t}$. This is because if the transmission probability is too small, the two devices are both in the listening state most of the time. If the transmission probability is too high, the two devices might miss the DA messages from each other because they are both in the transmitting state. It is also observed that, the handshake based ND protocol is superior to the one-way ND protocol in both DO mode and DD mode, which is as proved in proposition 1. Comparing Fig. 5 (a) and (b), we can also see that using the DD mode leads to a longer ND time than that of using the DO mode.

A two-device model for different D-ND protocols has been presented. However, it is not straightforward to extend the analytical model from two devices to a multiple devices model. To deduce a model for a D-ND process with multiple devices, we focus on the ND process of a target device instead of obtaining the ND time and ND ratio for all the devices within the network.

\section{B. D-ND Analysis for a Generic Model}

1) One-way DO-ND: To construct a scenario involving multiple devices, a circular full mesh network is used in this. Let the device $m$ be the target device, which is located at the center of the network. Let the node degree (the number of direct neighbors of the device in the network) of the target device be $k$, and the $k$ neighbors are uniformly distributed within this circular area. Using one-way DO-ND, device $m$ can detect its neighbors by receiving DA messages from them. As we mentioned before, at the beginning of each frame a device has a probability $p_{t}$ to be in the transmitting state and $1-p_{t}$ to be in the listening state. In a certain frame $i$, the probability that device $m$ is in the listening state and its $w$ neighbors are in the transmitting state is denoted as $p_{w}$, and is given by,

$$
p_{w}=\left(1-p_{t}\right)\left(\begin{array}{c}
k \\
w
\end{array}\right) p_{t}^{w}\left(1-p_{t}\right)^{k-w} .
$$

Let us denote $n_{i, x}$ as the number of DA messages that arrive at device $m$ in the $x^{t h}$ slot of the $i^{t h}$ frame, so we have $\sum_{x=1}^{N_{b}} n_{i, x}=w$. For a certain combination $\mathcal{N}_{i}=$ $\left[n_{i, 1}, \ldots, n_{i, N_{b}}\right], l_{i, z}$ is denoted as the number of elements, which are equal to $z$, in $\mathcal{N}_{i}$. For instance, if device $m$ receives three DA messages from two time slots within one round of scanning, $l_{i, 3}=2$. We have $\sum_{z=0}^{\max \left(\mathcal{N}_{i}\right)} l_{i, z}=N_{b}$.

Proposition 2: The probability to obtain a given $\mathcal{N}_{i}$ on the condition that all the elements in it appear in an arbitrary order is given by,

$$
p_{i}=\frac{w !}{\prod_{z=1}^{\max \left(\mathcal{N}_{i}\right)} l_{i, z} !} \frac{N_{b} !}{\prod_{x=1}^{N_{b}} n_{i, x} !} N_{b}^{-w} .
$$

Proof: For the proof please refer to Appendix A.

If multiple DA messages arrive in a time slot simultaneously $(z>1)$, these messages collide with each other and cannot be received by the receiver. Hence, the average number of received DA messages in one frame is given by,

$$
n_{r t}=\sum_{w=0}^{k} \sum_{i=1}^{\varpi} l_{i, 1} p_{i} p_{w}
$$

where, $\varpi$ is the total number of occupancy combinations of $\mathcal{N}_{i}$, which is a function decided by node degree $k$ and the number of slots in one frame $N_{b}$. The number of distinguishable occupancy of $\mathcal{N}_{i}$ is denoted as $A_{w, N_{b}}$, where $A_{w, N_{b}}=\left(\begin{array}{c}w+N_{b}-1 \\ N_{b}-1\end{array}\right)$ [26]. The relation between $A_{w, N_{b}}$ and $\varpi$ is given by,

$$
A_{w, N_{b}}=\sum_{i=1}^{\varpi} \frac{w !}{\prod_{z=1}^{\max \left(\mathcal{N}_{i}\right)} l_{i, z} !}
$$

Therefore, the probability for device $m$ to detect $k^{\prime}$ neighbors within consecutive $J$ frames is formulated as:

$$
P\left(J, k^{\prime}\right)=\sum_{j=0}^{k^{\prime}} \sum_{x=0}^{k^{\prime}-j} P\left(J-1, k^{\prime}-j\right) \rho(x+j) \frac{\left(\begin{array}{c}
k^{\prime}-j \\
x
\end{array}\right)\left(\begin{array}{c}
k-k^{\prime}+j \\
j
\end{array}\right)}{\left(\begin{array}{c}
k \\
x+j
\end{array}\right)},
$$

where, $\rho(x+j)$ is the probability of device $m$ receiving $x+$ $j$ DA messages within the $J^{t h}$ frame, in which $x$ messages are from known neighbors and $j$ messages are from unknown neighbors, and $\rho(v)=\sum_{w=1}^{k} \sum_{i=1}^{\varpi} 1_{\left(l_{i, 1}=v\right)} p_{i} p_{w} .1_{(\Omega)}$ is an indicator function, and it is equal to 1 if $\Omega$ is true, otherwise 0 .

2) Handshake based DO-ND: Using handshake based DOND, device $m$ can detect its neighbors in two scenarios: first, $m$ is in the listening state and receives DA messages from its neighbors; second, $m$ is in transmitting state and receives DA ACKs from its neighbors. We denote device $m$ receiving $n_{h}$ DA messages in a frame. The number $n_{h}$ can be expressed as $n_{h}=n_{r t}+n_{t r}$, where $n_{r t}$ and $n_{t r}$ are the number of DA messages received when device $m$ is in the listening state and transmitting state respectively. $n_{r t}$ can be obtained from (14), and $n_{t r}$ is derived here. When device $m$ is in the transmitting state, it sweeps its DA messages in all the possible directions. Once its neighbors receive the DA messages, they reply to it with DA ACK. Therefore, device $m$ could also discover its neighbors during the transmitting state. The number of received DA ACKs within a frame depends on the number of neighbors that reply to the DA messages in a certain slot. For the sake of simplicity in analysis, we assume that a device always replies to the received DA messages from its neighbors. Let $p_{x}$ be the probability for $x$ neighbors within a certain beam sector of device $m$, and $y$ out of these $x$ neighbors are in the listening state, where

$$
p_{x}=\left(\begin{array}{l}
k \\
x
\end{array}\right){\frac{1}{N_{b}}}^{x}\left(1-\frac{1}{N_{b}}\right)^{k-x}\left(\begin{array}{l}
x \\
y
\end{array}\right)\left(1-p_{t}\right)^{y} p_{t}^{x-y} .
$$

The condition for device $m$ to receive one DA ACK is that only one out of $y$ neighbors that are in the listening state replies to $m$, because if more than one neighbors reply to $m$ at the same time, DA ACKs will collide. The reason that the other $(y-1)$ neighbors cannot reply is because they do not correctly receive DA message from $m$, for instance, they 


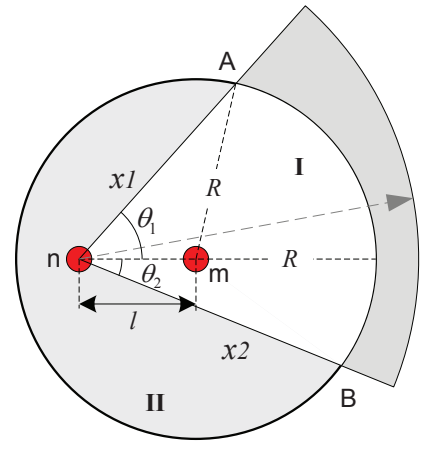

Fig. 6. Effective interfering area illustration.

receive more than one DA message at the same time from other neighbors, the probability of which is denoted by

$$
p_{y}=\left(\begin{array}{l}
y \\
1
\end{array}\right) p_{s}\left(1-p_{s}\right)^{y-1}
$$

where $p_{s}$ is the probability for a neighbor that is in the listening state and receives a DA message from device $m$ correctly in a certain time slot. For a device, say $n$, on the condition that $m$ is in the transmitting state and $n$ is in the listening state, $n$ receives DA message from device $m$ if there are no other devices transmitting in the direction of device $n$. Therefore, we have

$$
p_{s}=\left(1-\frac{p_{t}}{N_{b}}\right)^{k-x-1}\left(1-\frac{1}{N_{b}}\right)^{x-y} .
$$

Hence, the average number of received DA messages when device $m$ is in the transmitting state is

$$
n_{t r}=\sum_{x=1}^{k} \sum_{y=1}^{x} p_{t} p_{x} p_{y}
$$

3) One-way DD-ND: For the one-way DD-ND, all the devices only use directional mode for transmitting and receiving packets. At the beginning of a frame, if a device is in the listening state, it randomly chooses a beam sector to listen to. If multiple DA messages arrive at a device simultaneously, only the packets from the listening direction can be received. According to the definition given in Section IV-B1 and (14), the average number of received DA messages in one frame can be modified as

$$
n_{r t}=\left.\sum_{w=0}^{k} \sum_{i=1}^{\varpi} \sum_{x=1}^{N_{b}} 1\right|_{n_{i, x} \geq 1}\left(\begin{array}{c}
n_{i, x} \\
1
\end{array}\right)\left(1-\frac{1}{N_{b}}\right)^{n_{i, x}-1} \frac{1}{N_{b}} p_{i} p_{w},
$$

in which $n_{i, x}$ is the number of DA messages that arrive at device $m$ in the $x^{t h}$ slot of the $i^{t h}$ frame.

4) Handshake based $D D-N D$ : In the same way as that of handshake based DO-ND, devices can detect neighbors in both transmitting and listening state using handshake based DDND. When device $m$ is in the listening state, the number of received DA messages is obtained according to (21). Figure 6 is taken as an example, to explain how to deduce the number of received DA ACKs when device $m$ is in the transmitting state. Within a certain frame, we assume that device $m$ is in the transmitting state and one of its neighbors, say device $n$, is in the listening state and listens to the direction where device $m$ is covered as shown in Fig. 6. Being different from the handshake based DO-ND protocol, not all the devices that are in the transmitting state transmit in the direction of device $n$ and thus can cause DA collisions at device $n$. Because $n$ selects a certain direction to listen to and thus nullifies interference from other directions, only devices within listening direction of $n$ may interfere with the reception of the DA message from $m$. As shown in Fig. 6, the white area, $I$, is the intersection between the receiving sector of device $n$ and the circular network, which can be considered as the potential interfering area for device $n$. If other transmitting devices are located in this area, and they point in the direction of device $n$, then $n$ cannot receive a DA message from $m$ due to a DA collision. The average size of the potential interfering area is given by,

$$
\begin{aligned}
S & =\frac{1}{2} \int_{0}^{R} \int_{0}^{\theta}\left(l x_{1} \sin \theta_{1}+R^{2} \arcsin \left(\frac{x_{1} \sin \theta_{1}}{R}\right)\right. \\
& \left.+l x_{2} \sin \theta_{2}+R^{2} \arcsin \left(\frac{x_{2} \sin \theta_{2}}{R}\right)\right) f\left(\theta_{1}\right) f(l) d \theta_{1} d l
\end{aligned}
$$

where $R$ is the network radius. The summation of $\theta_{1}$ and $\theta_{2}$ is the antenna beamwidth $\theta$, and $l$ is the distance between device $m$ and device $n$. $f\left(\theta_{1}\right)$ and $f(l)$ are the distribution for the angle $\theta_{1}$ and distance $l$, where $f\left(\theta_{1}\right)=1 /(2 \pi)$ and $f(l)=2 l / R^{2}$. Receiving beam sector of device $n$ intersects with the circular network at point $A$ and point $B$. The distance from device $n$ to $A$ is $x_{1}$, and the distance from device $n$ to $B$ is $x_{2}$, where $x_{1}$ and $x_{2}$ are the solutions to the polynomial equations,

$$
\left\{\begin{array}{l}
x_{1}^{2}+l^{2}-R^{2}=2 \cos \theta_{1} x_{1} l \\
x_{2}^{2}+l^{2}-R^{2}=2 \cos \theta_{2} x_{2} l
\end{array}\right.
$$

In a certain time slot when device $m$ transmits a DA message to $n$, the condition for correct reception of this DA message by device $n$ is that device $n$ listens in the direction of $m$ and no other device within the listening direction of $n$ transmits in $n$ 's direction. The probability of this event is given by,

$$
p_{s}=\sum_{x=0}^{k-1} \frac{\left(\begin{array}{c}
k-1 \\
x
\end{array}\right)}{N_{b}}\left(\frac{S}{\pi R^{2}}\right)^{x}\left(1-\frac{p_{t}}{N_{b}}\right)^{x}\left(1-\frac{S}{\pi R^{2}}\right)^{k-x-1}
$$

When device $m$ is in the transmitting state, the expected number of DA ACKs received, $n_{t r}$, can be calculated according to (17), (18) and (20).

\section{NumERiCAL Results}

We verify the analysis employed thus far through simulations. According to the assumptions used in the analytical model, a two-dimensional circular network is formed in simulation scenarios, in which all the devices are within the transmission range of each other. The target device is located in the middle of the network, and all the other devices are uniformly distributed within the network. This means that the position as well as the direction of the antennas is also uniformly randomly distributed. The devices are assumed to 

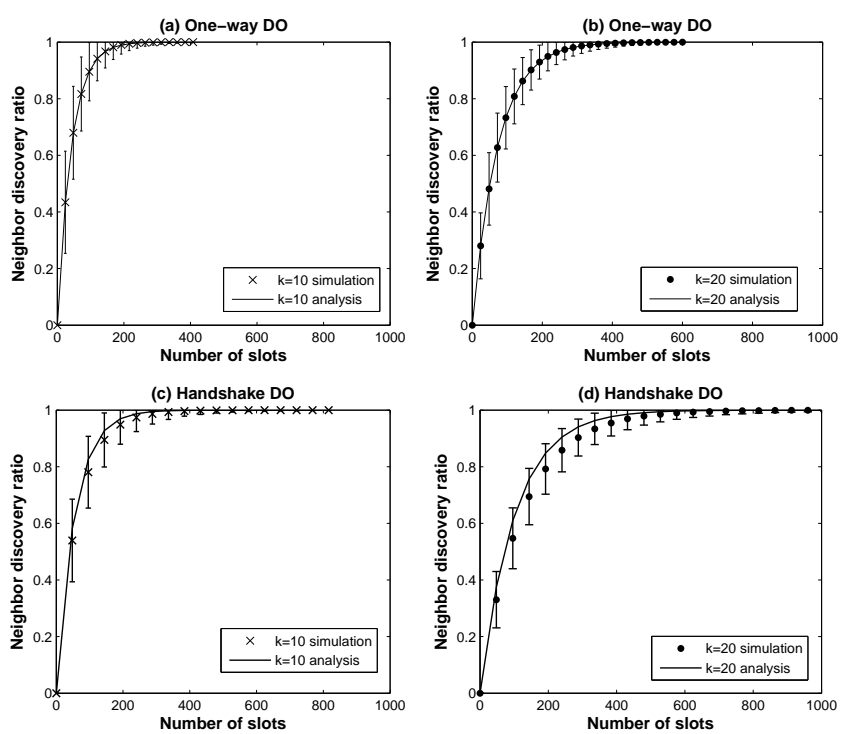

Fig. 7. Validation of the ND ratio of one-way and handshake based DO-ND through simulation, $\theta=60^{\circ}, p_{t}=0.3$.
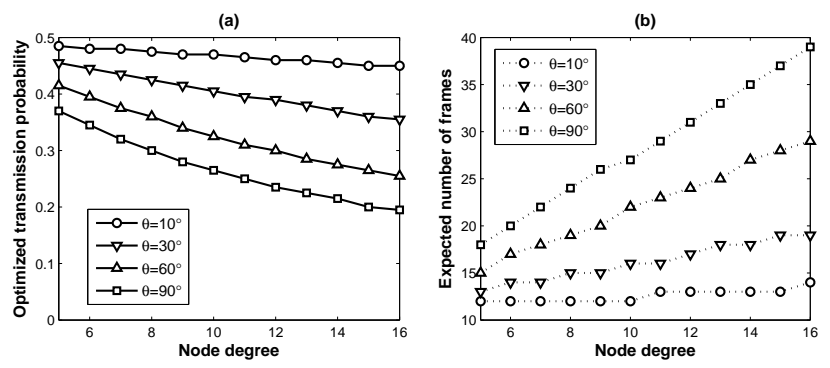

Fig. 8. Relationship between the node degree, optimized transmission probability and expected number of frames required for one ND process.

be equipped with flat-top antennas. The ND ratio mentioned in this section refers to the ND ratio of the target device in the middle of the network. All the simulation results are based on the average of 10000 iterations.

Figure 7 compares the performance of using one-way and handshake based D-ND mechanisms using DO mode when the number of neighbors is equal to 10 and 20 respectively. The simulation results are plotted with the mean and $95 \%$ confidence interval. These figures illustrate a good accuracy

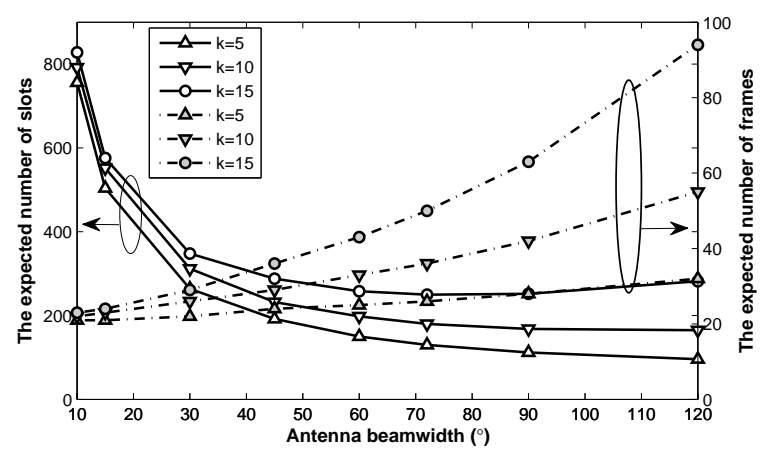

Fig. 9. Influence of antenna beamwidth on the ND performance, $p_{t}=0.3$.
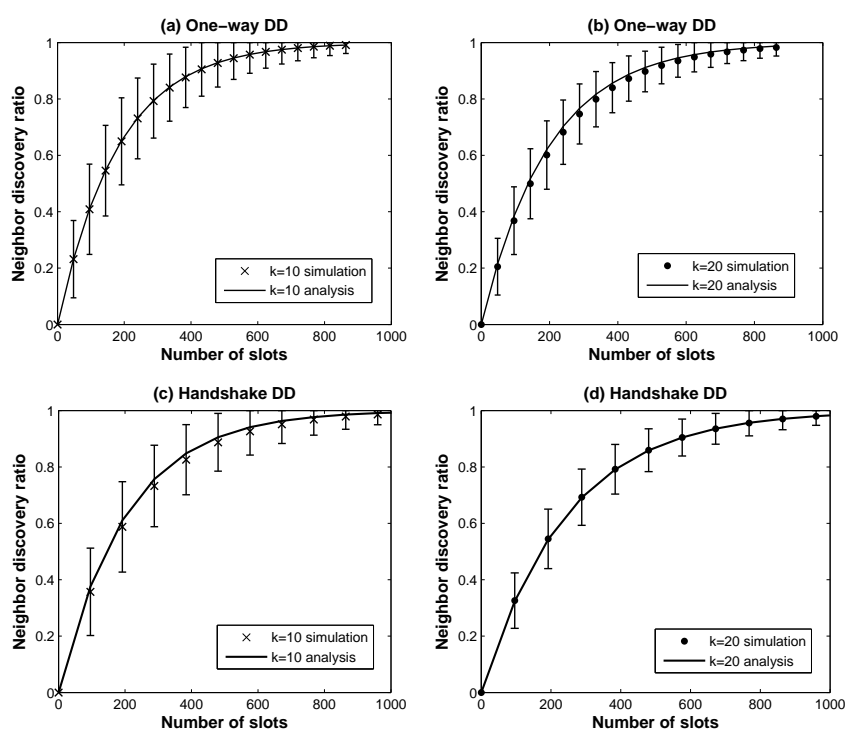

Fig. 10. Validation of the ND ratio of one-way and handshake based DD-ND through simulation, $\theta=60^{\circ}, p_{t}=0.3$.

of our proposed analytical model. From Fig. 7, it is observed that the handshake based mechanism performs worse than the one-way mechanism, which is different from the results of the two-device model. The reason that causes this phenomenon is: when multiple listening devices are in the same beam sector and transmit multiple DA ACKs simultaneously, collision of DA ACKs compromises the D-ND performance.

The optimal transmission probability $p_{\text {opt }}$ is obtained to maximize the number of detected neighbors in one frame. The relationship between the node degree and $p_{\text {opt }}$ is depicted in Fig. 8 (a). The empirical values of the optimal transmission probability can be used to achieve a better ND performance. For instance, in an IEEE 802.15.3c featured WPAN, once a device associates with the PNC of the WPAN, it is informed by the PNC the number of devices within the WPAN [1]. The device can use the obtained node degree of the WPAN to select an optimal transmission probability for neighbor discovery. In Fig. 8 (b), it shows the expected number of frames required for a ND process using a particular node degree and the optimal transmission probability. Here, the expected number of frames is also equivalent to the expected number of scans required for a ND process.

The influence of antenna beamwidth on the ND duration is shown in Fig. 9. Although the expected number of frames increases, with the increase in the antenna beamwidth, the entire ND time, which is related to the number of expected slots used for a ND process decreases. The analytical results for the ND ratio obtained using one-way DD-ND and handshake based DD-ND are depicted in Fig. 10, which are compared with the results from simulations. These two figures also indicate a good accuracy of our proposed analytical model.

To obtain an overview, in Fig. 11, the four D-ND protocols, that is, one-way DO, one-way DD, handshake DO, and handshake DD, are compared together in terms of ND ratios using simulations. The node degree is set to 10 and the transmission probability is set to 0.3 . Many interesting aspects are observed 

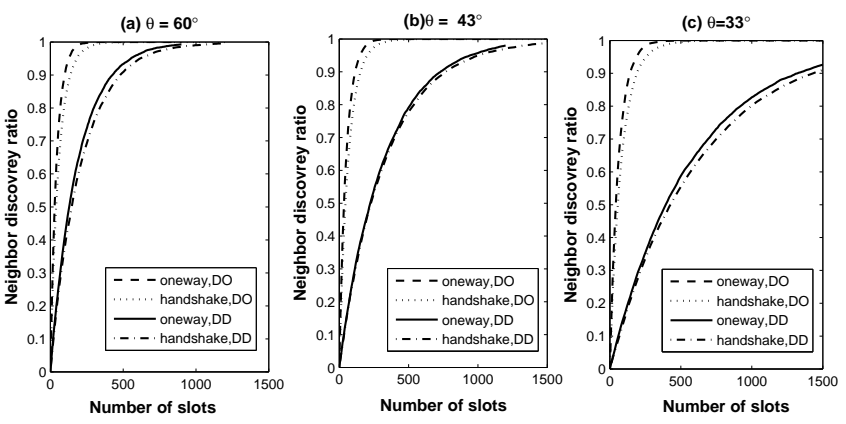

Fig. 11. Comparison of ND ratio using flat-top directional antennas, $k=10$, $p_{t}=0.3$.

in Fig. 11:

- In general, using DO mode has higher ND ratio than using DD mode.

- When the antenna beamwidth decreases, the ND performance difference between using DO mode and DD mode increases.

- When antenna mode is fixed (DO or DD), the one-way mechanism has a higher ND ratio than the handshake based mechanism.

In this article, we assumed that the antenna system has the capability to scan $360^{\circ}$. However in practice it is not true. For instance, in the patch-based antenna system the typical scan range is around $90^{\circ}$, which means that for such antennas the number of beam sectors is less than the antennas that can scan omni-directionally. Correspondingly this kind of antennas has fewer beam sectors and less number of neighbors, which might reduce the neighbor discovery duration compared with the device that can scan omnidrectionally. Therefore the results presented here provide an upper bound on the neighbor discovery time and for the worst case scenario.

\section{Antenna Pattern And Link Model Influence on ND PERFORMANCE}

To illustrate the influence of antenna pattern on the performance of ND processes, simulations are carried out using a UCA antenna system with LM2 link model to compare with the results obtained using the flat-top antenna system with LM1 link model on the condition that all the devices are within the transmission range of each other.

\section{A. ND Ratio}

In Fig. 12, one-way DO, handshake based DO, one-way DD, and handshake based DD are compared together using UCA antenna systems with different number of antenna elements. In Fig. 12, it is observed that the DD mode performs better than the DO mode in all the scenarios, which is different from the results as shown in Fig. 11. This is because, the UCA and the flat-top systems have a large difference in terms of the antenna gain pattern due to the influence of side lobes, which is shown in Fig. 3. Note that the antenna beamwidth used for these two antenna patterns are similar here. For the UCA system, the side lobes play an important role in
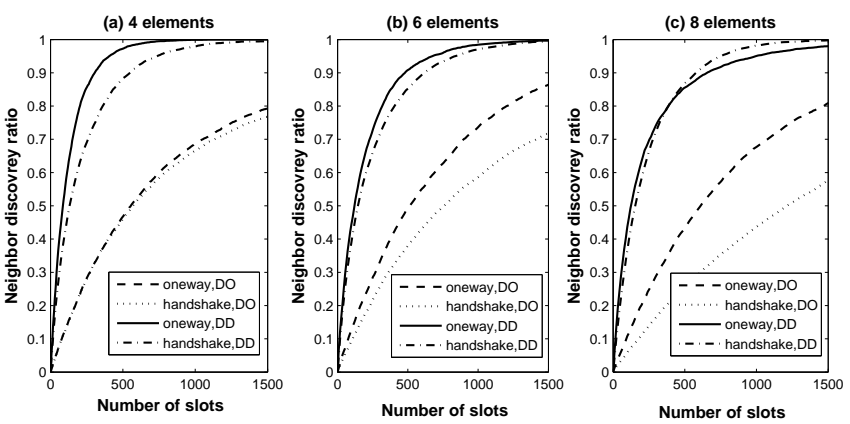

Fig. 12. The influence of the number of antenna elements of UCA systems on ND ratio with $p_{t}=0.3$, path loss exponent $n=2.5, k=10, R=5 \mathrm{~m}$.

the D-ND process. If a side lobe has sufficient gain, it can also be used to discover a neighbor that is not pointed by the main lobe. Meanwhile, a side lobe may also generate interference to disturb the transmissions amongst other links, if the interference is high enough. The performance between one-way and handshake based mechanisms is very close for DD mode with a 6-element UCA system and using DO mode with an 8-element UCA system. Except these two scenarios, one-way mechanism performs better than handshake based mechanism and this can also be observed in Fig. 11.

\section{B. ND Overhead}

In Fig. 13 (a) and (b), the total amount of generated DA and DA ACK messages, which are also defined as overhead, is compared using different D-ND mechanisms using the UCA system and the flat-top system respectively, in which the results show the mean with $95 \%$ confidence interval. When the flat-top system is used in the simulations, the following properties are observed. Firstly, the amount of overhead increases with the decrease of antenna beamwidth in all the D-ND mechanisms. Secondly, using DD mode generates more overhead than using DO mode for both oneway and handshake based mechanisms. Thirdly, using DO mode, handshake based mechanism generates slightly more overhead than one-way mechanism. Fourthly, using DD mode, the amount of overhead generated using one-way mechanism is higher than using handshake based mechanism.

To explain the above observations, the expected numbers of scanning using the UCA system and the flat-top system are plotted in Fig. 13 (c) and (d). Although the expected ND time obtained using one-way mechanism is faster than using handshake based mechanism shown in Fig. 11, the expected number of scanning using one-way mechanism is higher than the handshake based mechanism as shown in Fig. 13 (d). The expected number of scanning relates to the number of DA messages transmitted during a ND process. As shown in Fig. 13 (d), when the DO mode is used, one-way and handshake based mechanisms have similar number of expected scanning, which implies that the number of generated DA messages are also similar. For the handshake based mechanism, other than DA messages, it also generates DA ACK messages. This leads to increase in overhead compared to the one-way DO. When the DD mode is used, the expected number of 

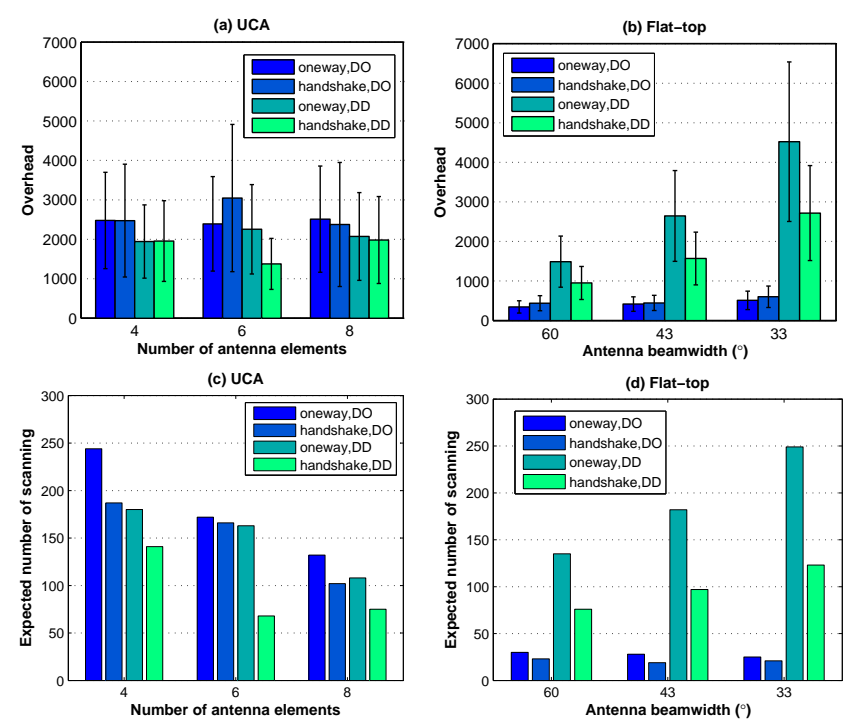

Fig. 13. Comparison between UCA and flat-top system on overhead and expected number of scanning with $p_{t}=0.3, k=10, n=2.5, R=5 \mathrm{~m}$.

scanning obtained using one-way mechanism is much higher than the handshake based mechanism. Hence the generated DA messages dominate the amount of overhead.

Compared to the flat-top systems, the overhead using UCA systems does not change a lot with the change of the number of antenna elements. Moreover, contrary to this, in the flattop systems the expected number of scanning decreases with the decrease in antenna beamwidth. This is because of the influence of antenna side lobes. As shown in Fig. 3 (a), when the number of antenna elements is less, the influence of the side lobes is higher, which decreases the D-ND performance due to the interference generated from the side lobes within a ND process. It is also seen that the one-way mechanism results in a higher number of scanning compared to the handshake based mechanism.

\section{Influence of $p_{t}$ and $k$}

The influence of transmission probability, $p_{t}$, and the node degree, $k$, on the expected number of scanning are depicted in Fig. 14, in which a 6-element UCA system is used in the simulation. The optimal transmission probabilities for the D-ND processes can be observed in Fig. 14 (a). Compared to Fig. 8 (a), the optimal transmission probability obtained using the UCA system is lower than the flat-top system with the same antenna beamwidth. This is also because of the interference generated by side lobes. As shown in Fig. 14 (b), the expected number of scanning increases with the increase in node degree, and the expected number of scanning obtained using handshake DD-ND is the lower bound compared to other mechanisms.

\section{CONCLUSION}

In a $60 \mathrm{GHz}$ radio wireless network, directional neighbor discovery (D-ND) is an important process to acquire the orientation information of the devices within a network for setting
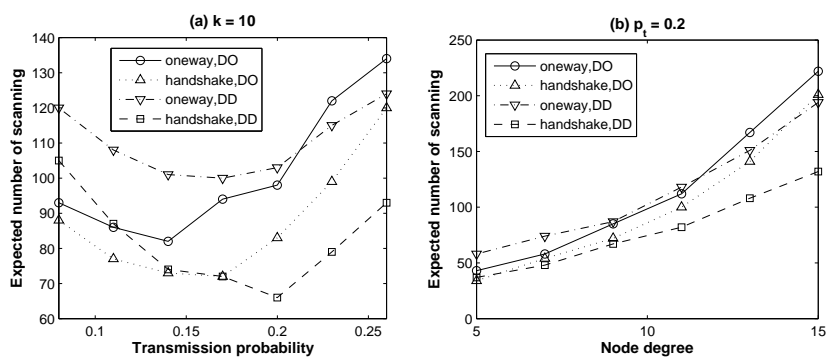

Fig. 14. Influence of transmission probability on the expected number of scanning using 6-element UCA system.

up directional links. In this work we thoroughly investigated the performance of ND processes using directional antennas. The main contribution of our work is two-folds: (i) a comprehensive analytical model was presented to demonstrate the ND performance considering different ND mechanisms (one-way ND and handshake based ND) as well as different antenna modes such as, directional transmitting omnidirectional receiving (DO) and directional transmitting directional receiving (DD) modes. Our analytical model is based on the flat-top antenna pattern and full mesh networks. We demonstrated the performance of these ND protocols in terms of ND ratio and ND time. We showed that, in a two-device scenario, the handshake based ND protocol outperforms the one-way ND protocol. However, in a generic scenario in which more than two devices are considered, the one-way ND protocol performs better than the handshake-based ND. Moreover, we also demonstrated that with the decrease in antenna beamwidth the duration of a ND process is prolonged. Our analytical model can be used to construct general guidelines for using directional antennas in wireless personal area networks. (ii) We studied the influence of the antenna pattern and the link model on D-ND processes. To obtain a detailed view of D-ND process using $60 \mathrm{GHz}$ radio, the D-ND performance was studied using a UCA system with an interference-aware link model via simulations. The results show that there are indeed some deviations between the analytical results and the realistic scenarios, which is due to the effect of side lobes in a UCA system. With more sophisticated antenna design, the effect of side lobes can be reduced. We argue it is worth to investigate more accurate analytical models using realistic antenna patterns in future work.

\section{APPENDIX A \\ OcCupancy Problem in Directional NeIGHbor DISCOVERY}

In one round of scanning, if device $m$ is in the listening state, the number of DA messages that arrive at $m$ in the $x^{t h}$ slot of one frame is denoted as $n_{x}, n_{1}+n_{2}+\ldots+n_{N_{b}}=w$, where $N_{b}$ is the number of beam sectors which is also equal to the number of slots in one frame, and $w$ is the number of neighbors that are in the transmitting state. This problem is an occupancy problem that randomly place indistinguishable $r$ balls into $n$ cells, where $r_{1}+r_{2}+\ldots+r_{n}=r$, and the number of elements in one cell can be zero. The number of 
ways to separate $r$ elements into $n$ sub-groups is given by [26]

$$
\begin{aligned}
& \left(\begin{array}{c}
r \\
r_{1}
\end{array}\right)\left(\begin{array}{c}
r-r_{1} \\
r_{2}
\end{array}\right)\left(\begin{array}{c}
r-r_{1}-r_{2} \\
r_{3}
\end{array}\right) \ldots\left(\begin{array}{c}
r-r_{1}-\ldots-r_{n-2} \\
r_{n-1}
\end{array}\right)\left(\begin{array}{c}
r_{n} \\
r_{n}
\end{array}\right) \\
= & \frac{r !}{r_{1} !\left(r-r_{1} !\right)} \frac{\left(r-r_{1}\right) !}{r_{2} !\left(r-r_{1}-r_{2} !\right)} \\
& \times \frac{\left(r-r_{1}-r_{2}\right) !}{r_{3} !\left(r-r_{1}-r_{2}-r_{3} !\right)} \ldots \frac{\left(r-r_{1}-\ldots-r_{n-2}\right) !}{r_{n-1} !\left(r-r_{1}-r_{2}-\ldots-r_{n-1} !\right)} \\
= & \frac{r !}{r_{1} ! r_{2} ! \ldots r_{n} !} .
\end{aligned}
$$

Let us denote $w$ as the maximum number of balls in one cell, where $w=\max \left(r_{n}\right)$. Therefore, we have $n_{0}+n_{2}+\ldots+n_{w}=$ $n$, where $n_{i}$ is the number of cells which contain $i$ balls in them, $0 \leq i \leq w$. According to (25), the number of ways to participate in cells is given by $\frac{n !}{n_{0} ! n_{1} ! \ldots n_{w} !}$. Therefore, the total number of distributions of the occupancy number with $\mathcal{N}_{n}=\left[r_{1}, r_{2}, \ldots, r_{n}\right]$ is given by,

$$
\mathbb{N}_{r, n}=\frac{r !}{r_{1} ! r_{2} ! \ldots r_{n} !} \times \frac{n !}{n_{0} ! n_{1} ! \ldots n_{w} !} .
$$

In total, there are $n^{r}$ possible placements which are equally probable, hence the probability $p$ to obtain the given occupancy number $\mathcal{N}_{n}$ is $p=\mathbb{N}_{r, n} n^{-r}$.

\section{ACKNOWLEDGMENT}

This work was supported by IOP GenCom FHN and the SiGiSpot projects in The Netherlands. Moreover, we would like to thank Dajie Liu, who provided valuable comments on this work.

\section{REFERENCES}

[1] IEEE 802.15 WPAN Millimeter Wave Alternative PHY TG3c., Std. [Online]. Available: http://www.ieee802.org/15/pub/TG3c.html

[2] J. Wang, Z. Lan, C. W. Pyo, T. Baykas, C. Sum, M. A. Rahman, J. Gao, R. Funada, F. Kojima, H. Harada, and S. Kato, "Beam codebook based beamforming protocol for multi-Gbps millimeter-wave WPAN systems," IEEE Journal on Selected Areas in Communications, 2009.

[3] Y. Ko, V. Shankarkumar, and N. H. Vaidya, "Medium access control protocols using directional antennas in ad hoc networks," in Proc. of IEEE INFOCOM, vol. 1, March 2000, pp. 13-21.

[4] S. Yi, Y. Pei, and S. Kalyanaraman, "On the capacity improvement of ad hoc wireless networks using directional antennas," in Proc. of the 4th ACM international symposium on MOBIHOC, 2003, pp. $108-116$.

[5] R. Ramanathan, "On the performance of ad hoc networks with beamforming antennas," in Proc. of the 2th ACM international symposium on MOBIHOC, 2001, pp. $95-105$.

[6] R. R. Choudhury, X. Yang, R. Ramanathan, and N. Vaidya, "Using directional antennas for medium access control in ad hoc networks," in Proc. of the 8th annual International Conference on Mobile Computing and Networking (MobiCom'02), Atlanta, Georgia, USA, 2002, pp. 59 70.

[7] K. Sohrabi, J. Gao, V. Ailawadhi, and G. J. Pottie, "Protocols for selforganization of a wireless sensor network," IEEE Personal Communications, vol. 7, no. 5, pp. 16-27, Oct. 2000.

[8] S. Vasudevan, J. Kurose, and D. Towsley, "On neighbor discovery in wireless networks with directional antennas," in Proc. of IEEE INFOCOM, vol. 4, March 2005, pp. 2502-2512.

[9] L. Galluccio, G. Morabito, and S. Palazzo, "Analytical evaluation of a tradeoff between energy efficiency and responsiveness of neighbor discovery in self-organizing ad hoc networks," IEEE Journal on Selected Areas in Communications, vol. 22, no. 7, pp. 1167-1182, September 2004.

[10] M. J. McGlynn and S. A. Borbash, "Birthday protocols for low energy deployment and flexible neighbor discovery in ad hoc wireless networks," in Proc. of the 2th ACM international symposium on MOBIHOC, October 2001, p. 137145.
[11] S. Singh, F. Ziliotto, U. Madhow, E. M. Belding, and M. Rodwell, "Blockage and directivity in $60 \mathrm{GHz}$ wireless personal area networks: From cross-layer model to multihop MAC design," IEEE Journal on Selected Areas in Communications, Special Issue on Realizing Gbps Wireless Personal Area Networks, 2009.

[12] Z. Zhang, "Performance of neighbor discovery algorithms in mobile ad hoc self-configuring networks with directional antennas," in MILCOM 2005, Oct. 2005, pp. 1-7.

[13] J. Ning, T. S. Kim, and S. V. Krishnamurthy, "Directional neighbor discovery in $60 \mathrm{GHz}$ indoor wireless networks," in Proc. of the 12th ACM international conference on Modeling, analysis and simulation of wireless and mobile systems (MSWiM'09), 2009.

[14] F. Yildirim and H. Liu, "A cross-layer neighbor-discovery algorithm for directional 60-ghz networks," IEEE Trans. on Vehicular Technology, vol. 58, pp. 4598-4604, 2009.

[15] Wireless Medium Access Control (MAC) and Physical Layer (PHY) Specifications for High Rate Wireless Personal Area Networks (WPANs), IEEE. Std. 802.15.3-2003 Std.

[16] R. Schmidt, "Multiple emitter location and signal parameter estimation," IEEE Trans. Antennas Propagation, vol. AP-34, pp. 276-280, March 1986.

[17] R. Roy and T. Kailath, "ESPRIT-estimation of signal parameters via rotational invariancetechniques," IEEE Transactions on Acoustics, Speech and Signal Processing, vol. 37, pp. 984-995, 1989.

[18] C. A. Balanis, Antenna theory : analysis and design. WileyInterscience, 2005.

[19] P. Smulders, "Statistical characterization of 60-ghz indoor radio channels," IEEE Transactions on Antennas and Propagation, vol. 57, no. 10, pp. 2820-2829, 2009.

[20] H. Yang, P. F. M. Smulders, and M. H. A. J. Herben, "Channel characteristics and transmission performance for various channel configurations at $60 \mathrm{GHz}$," EURASIP Journal on Wireless Communications and Networking, 2007.

[21] P. F. Smulders, "Exploiting the $60-\mathrm{GHz}$ band for local wireless multimedia access: Prospects and future directions," IEEE Communications Magazine, vol. 40, no. 1, pp. 140-147, Jan. 2002.

[22] R. Hekmat, Ad-hoc Networks: Fundamental Properties and Network Topologies. Springer, Oct. 2006.

[23] A. A. Abu-Dayya and N. C. Beaulieu, "Outage probabilities in the presence of correlated log-normal interferers," IEEE Transactions on Vehicular Technology, vol. 43, pp. 164-173, 1994.

[24] L. Fenton, "The sum of log-normal probability distributions in scatter transmission systems," IRE Transactions on Communications Systems, pp. 57-67, 1960.

[25] M. Abramowitz and I. A. Stegun, Eds., Handbook of Mathematical Functions. Dover Publications, Inc., New York, 1968.

[26] W. Feller, An Introduction to Probability Theory and its Applications, 3rd ed. John Wiley and Sons, 1968, vol. 1.

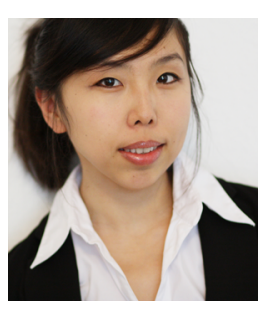

Xueli An received the Bachelor of Engineering degree from Harbin Institute of Technology, China, in July 2003. She received the M.Sc degree in Electrical Engineering from Delft University of Technology, The Netherlands, in July 2005. In Sept. 2006, she started her research work as a $\mathrm{PhD}$ candidate at the Wireless and Mobile Communications group, faculty of Electrical Engineering, Mathematics and Computer Science, Delft University of Technology, The Netherlands. From March 2009 to June 2009, she was a visiting researcher in the Ubiquitous Mobile Communications Group, National Institute of Information and Communications Technology (NICT), Yokosuka, Japan. Her PhD research focused on the medium access control layer and network layer design for $60 \mathrm{GHz}$ featured wireless personal area networks. Moreover, her research interests also involve ad-hoc and sensor networks, next-generation radio access networks, etc. Since November 2010, she joins Services Infrastructure Domain, Bell Labs, Antwerp, Belgium. 


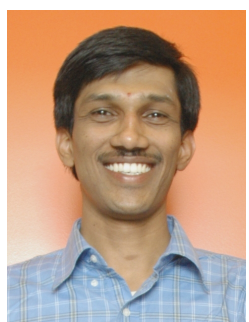

R. Venkatesha Prasad (M'95) received the bachelors degree in electronics and communication engineering and the M.Tech. degree in industrial electronics from the University of Mysore, Mysore, India, in 1991 and 1994, respectively, and the Ph.D. degree from Indian Institute of Science (IISc) Bangalore, India, in 2003. In 1994 and 1996, he was a Consultant and Project Associate for ERNET Laboratory of Electronics and Communication Engineering, IISc. From 1999 to 2003, he was a Consultant for CEDT, IISc, for VoIP application developments, as part of the Nortel-Networks-sponsored project. From 2003 to 2005, he headed a team of engineers at the Esqube Communication Solutions Pvt. Ltd. for the development of various real-time networking applications. Since 2005, he has been with theWireless and Mobile Communications Group, Department of Electrical Engineering, Mathematics and Computer Science, Delft University of Technology, Delft, The Netherlands, working on the European Union funded projects MAGNET/MAGNET Beyond and PNP 2008, IOPGencom FHN, and guiding post graduate students. He is also a Consultant for Esqube on a part-time basis. Dr. Prasad is a member of the IEEE SCC41 Standardization Committee. He has been a Cofounder of CogNet, Pernets, and E2Nets workshops. He has been a TPC member and an organizing committee member of many major workshops and conferences and he has chaired some of the conference committees and sessions. He has been a regular reviewer of many journals including many IEEE transactions and national project proposals. He has been part of four IEEE and ECMA standards and has co-authored 100 publications including a few patents. He is also an invited member of IEEE ComSoc Standards Board as a liaison of IEEE Comsoc TCCC.

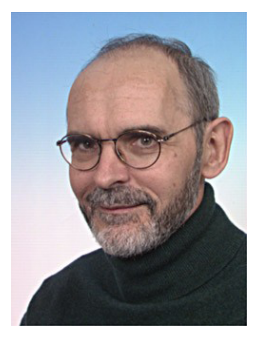

Ignas G. M. M. Niemegeers got a degree in Electrical Engineering from the University of Ghent, Belgium, in 1970. In 1972 he received a M.Sc.E. degree in Computer Engineering and in 1978 a Ph.D. degree from Purdue University in West Lafayette, Indiana, USA. From 1978 to 1981 he was a designer of packet switching networks at Bell Telephone Mfg. Cy, Antwerp, Belgium. From 1981 to 2002 he was a professor at the Computer Science and the Electrical Engineering Faculties of the University of Twente, Enschede, The Netherlands. From 1995 to 2001 he was Scientific Director of the Centre for Telematics and Information Technology (CTIT) of the University of Twente, a multi-disciplinary research institute on ICT and applications. Since May 2002 he holds the chair Wireless and Mobile Communications at Delft University of Technology, where he is heading the Telecommunications Department. He was involved in many European research projects, e.g., the EU projects MAGNET and MAGNET Beyond on personal networks, EUROPCOM on UWB emergency networks and, eSENSE and CRUISE on sensor networks. He is a member of the Expert group of the European technology platform eMobility and IFIP TC-6 on Networking. His present research interests are $4 \mathrm{G}$ wireless infrastructures, future home networks, ad-hoc networks, personal networks and cognitive networks. He has (co)authored close to 300 scientific publications and has coauthored a book on Personal Networks. 